Sección Agrícola / Agriculture

Artículos de investigación / Research paper

\title{
Modelización espacial de trips (Insecta: Thysanoptera) en el cultivo de aguacate (Persea americana)
}

\author{
Spatial modeling thrips (Insecta: Thysanoptera) in growing avocado (Persea americana)
}

\author{
ROBERTO RIVERA-MARTÍNEZ1, JOSÉ FRANCISCO RAMÍREZ-DÁVILA², MARTÍN RUBÍ-ARRIAGA², \\ AURELIO DOMÍNGUEZ-LÓPEZ ${ }^{3}$, AGUSTÍN DAVID ACOSTA-GUADARRAMA ${ }^{1}$ \\ y DULCE KAREN FIGUEROA-FIGUEROA ${ }^{1}$
}

\begin{abstract}
Resumen: México es el principal productor y exportador de aguacate en el mundo. Los trips son considerados una de las principales plagas de este cultivo, ya que se alimentan del fruto pequeño y forman crestas o protuberancias, las cuales al crecer el fruto son más visibles, y este pierde valor económico. Además de este daño, las heridas causadas por los trips son el principal causante para que entre la enfermedad denominada roña de fruto. Las alternativas de control de los trips han carecido de eficacia debido, entre otras causas, a que se desconoce su distribución espacial dentro de las huertas de aguacate. Este trabajo tuvo por objetivo determinar la distribución espacial de las poblaciones de trips en aguacate mediante el uso de técnicas de estadística espacial que condujeron a la generación de mapas por medio del "krigeado". Los resultados demostraron que las poblaciones de trips presentan una distribución de tipo agregada, que fue corroborada por los mapas de densidad. Las infestaciones se distribuyeron en el $100 \%$ de la superficie de las dos parcelas experimentales, lo cual resulta interesante para dirigir las medidas de control sobre áreas específicas de infestación. Se logró determinar estabilidad espacial y temporal a corto plazo de las poblaciones de trips.
\end{abstract}

Palabras clave: Geoestadística, krigeado, mapas de densidad.

\begin{abstract}
Mexico is the main producer and exporter of avocados in the world, the trips are considered a major pest of avocado in Mexico, as they feed on small fruit and form ridges or bumps which as the fruit grow they are more visible and it loses economic value. In addition to this damage, injuries caused by thrips are the main cause for presence of avocado scab. Alternative control against thrips has lacked effectiveness because their spatial distribution is unknown in avocado orchards. This study aimed to determine the spatial distribution of populations of thrips in avocado using spatial statistical techniques that led to the generation of maps through kriging. The results showed that populations of thrips present an aggregate type of distribution, which was corroborated by density maps. Infestations were distributed in 100 $\%$ of the two experimental plots, which is interesting to apply control measures only on specific areas of infestation. It was possible to determine spatial and temporal short-term stability thrips populations.
\end{abstract}

Key words: Geoestatistics, kriging, density maps.

\section{Introducción}

El aguacate (Persea americana Mill.) es la cuarta fruta más importante en el mundo. Su producción mundial se estima en 4,2 millones de toneladas, siendo México el principal productor con una producción promedio anual de 1107 135,16 ton en 134 322,12 ha (SIAP 2014).

La producción nacional de aguacate en México ha mostrado una tendencia creciente en superficie cultivada y volumen de producción, con predominio de la variedad Hass, la cual destaca por su demanda a nivel mundial (Naamani 2007). En México, el principal productor es Michoacán, seguido por Nayarit, Morelos y México (SIAP 2014).

El cultivo del aguacate presenta una gran diversidad de plagas entre las que se encuentran: trips (Heliothrips haemorrhoidalis Bouché), los barrenadores pequeño y grande de la semilla (Conotrachelus persea Barber y Helipus lauri Boheman), la palomilla barrenadora de la semilla (Stenoma catenifer Walshingam), el barrenador del tronco y ramas (Copturus aguacatae Kissinger), las arañas café (Oligonichus punicae Hirst) y cristalina (O. perseae Tuttle), entre otras más (Coria 1993).

Los trips (Thysanoptera) han adquirido importancia para el aguacate dado que, estos insectos se alimentan del fruto pequeño (Salgado 1993) y forman crestas o protuberancias sobre el pericarpio (González et al. 2000). Las deformaciones son más visibles cuando el fruto madura (Fisher y Davenport 1989; González et al. 2000) lo que ocurre muy probablemente con larvas y adultos de Frankliniella y Neohydatothrips. Sin embargo, en las especies de Scirtothrips, las larvas y adultos causan daños en follaje tierno, provocando distorsión y cicatrices castaño oscuro a lo largo de la nervadura central de la hoja, en el envés, esto aunado al daño que causan en los frutos. Algo similar ocurre con el fruto joven, que puede que-

\footnotetext{
${ }^{1}$ Estudiante Programa de Doctorado en Ciencias Agropecuarias y Recursos Naturales. Universidad Autónoma del Estado de México, UAEM. Toluca, México CP 50200.rob_m@live.com.mx. ${ }^{2}$ Director del Laboratorio de Investigaciones Entomológicas y Tecnologías en Agricultura de Precisión; Facultad de Ciencias Agrícolas, UAEM. Cerrillo Piedras Blancas s/n km 15 Carr. Toluca - Ixtlahuaca, entronque al Cerrillo. C.P. 50200; Toluca, México.jframirezd@uaemex.mx, autor para correspondencia. ${ }^{3}$ Profesor-Investigador Facultad de Ciencias Agrícolas, UAEM. Cerrillo Piedras Blancas s/n km 15 Carretera Toluca - Ixtlahuaca, entronque al Cerrillo. C.P. 50200; Toluca, México.
} 
dar completamente cubierto por una cicatriz castaño-oscura, aunque comúnmente se observan cicatrices pequeñas, a veces alargadas, este daño es atribuido a Scirtothrips perseae Nakahara (Hoddle 2002). Ascensión et al. (1999) y Johansen et al. (1999) afirmaron que este síntoma asociado a trips aparece en el fruto pequeño, aunque no se ha precisado las etapas fenológicas más afectadas.

Las densidades de trips en huertos de aguacate se han incrementado año tras año y el control químico ha sido poco eficiente en el manejo de sus poblaciones. La infestación de este insecto plaga, hace importante implementar actividades que permitan conocer el impacto real que tiene sobre el cultivo de aguacate $\mathrm{y}$, con base en esta información, aplicar medidas de control eficaces contra la plaga. Por lo tanto, el objetivo de este trabajo fue determinar la distribución espacial de las poblaciones de trips en aguacate mediante el uso de técnicas geoestadísticas.

\section{Materiales y métodos}

El estudio se llevó a cabo en una zona productora de aguacate en la región de Coatepec Harinas del Estado de México, donde se seleccionaron dos parcelas comerciales de aguacate de la variedad Hass de aproximadamente cinco años de edad. Cada parcela constó de cuatro hectáreas y se dividió en cuadrantes de $20 \times 20 \mathrm{~m}$, para tener un total de 100 cuadrantes por parcela. Bajo el método de muestreo por cuadrantes, se tomaron 40 al azar y por cada uno se seleccionaron tres árboles, para un total de 120 árboles en cada parcela experimental.

En las dos parcelas se realizaron muestreos quincenales durante 10 meses y en cada uno se contó el número de trips atrapados en trampas amarillas. Cada árbol muestreado fue georreferenciado para obtener sus coordenadas.

Se emplearon trampas amarillas consistentes en vasos de plástico del número ocho marca $\mathrm{JAGUAR}^{\odot}$, de $10 \mathrm{~cm}$ de alto, $4,5 \mathrm{~cm}$ de diámetro en la base y 7,5 cm de diámetro en el borde superior y de color amarillo; de acuerdo con lo sugerido por González et al. (1998) a cada vaso se le puso un gancho en la base y un adherente (manteca vegetal) en la superficie externa. Se colocaron cuatro trampas en los árboles en cada punto cardinal que fueron revisadas quincenalmente para el conteo y retiro de los trips capturados; posteriormente, se volvían a colgar en el árbol. Algunos especímenes de trips fueron guardados en alcohol etílico al 70 $\%$, posteriormente, se montaron en portaobjetos y se identificaron mediante claves a nivel de especie, en el Laboratorio de Entomología de la Universidad Nacional Autónoma de México.

Análisis geoestadístico. Se obtuvo el semivariograma experimental a partir del valor promedio mensual de trips capturados en cada muestreo semanal utilizando el programa variowin 2.2 (Software for spatial data analysis en 2D. New York, EEUU). El valor experimental del semivariograma fue calculado con la siguiente expresión (Journel y Huijbregts 1978; Isaaks y Srivastava 1989):

$$
\boldsymbol{\gamma}^{*}(\mathbf{h})=\frac{1}{2 \mathrm{~N}(\mathrm{~h})} \sum_{i=1}^{\mathrm{N}(\mathrm{h})}\left[z\left(x_{i}+\mathrm{h}\right)-z\left(x_{i}\right)\right]^{2}
$$

dónde: $\gamma^{*}(\mathrm{~h})$ es el valor experimental del semivariograma para el intervalo de distancia $\mathrm{h}$; $\mathrm{n}(\mathrm{h})$ es el número de pares de puntos mues- trales separados por el intervalo de distancia $\mathrm{h} ; \mathrm{z}\left(\mathrm{x}_{\mathrm{i}}\right)$ es el valor de la variable de interés en el punto muestral $x_{i}$ y $z\left(x_{i}+h\right)$ es valor de la variable de interés en el punto muestra $x_{i}+h$.

Estimación de los parámetros del modelo de semivariograma. Una vez obtenido el semivariograma experimental, se realizó su ajuste a algún semivariograma teórico (esférico, exponencial, gaussiano, etc.) Englund y Sparks (1988) utilizando el programa Variowin versión 2.2.

Validación del modelo teórico. Se siguió un procedimiento de validación cruzada, en el cual los parámetros del modelo Co (efecto de pepita), C + Co (meseta) y a (rango o alcance) se van ajustando de forma interactiva (prueba y error) hasta obtener los mejores valores de los estadísticos:

a. Media de los errores de estimación (MEE):

$$
\mathbf{M E E}=\frac{1}{n} \sum_{i=1}^{n}\left[z^{*}\left(x_{i}\right)-z\left(x_{i}\right)\right]
$$

dónde: $\mathrm{z}^{*}\left(\mathrm{x}_{\mathrm{i}}\right)$ es el valor estimado de la variable de interés en el punto $\mathrm{x}_{\mathrm{i}} ; \mathrm{z}\left(\mathrm{x}_{\mathrm{i}}\right)$ es el valor medido de la variable de interés en el punto $\mathrm{x}_{\mathrm{i}}$ y $\mathrm{n}$ es el número de puntos muéstrales utilizado en la interpolación. La MEE no debe ser significativamente distinta de 0 (prueba de t), en cuyo caso, indicaría que el modelo de semivariograma permite el cálculo de estimadores no sesgados.

b. Error cuadrático medio (ECM):

$$
\mathbf{E C M}=\frac{1}{n} \sum_{i=1}^{n}\left[z^{*}\left(x_{i}\right)-z\left(x_{i}\right)\right]^{2}
$$

Un modelo de semivariograma se considera adecuado si, como regla práctica, el valor del estadístico es cercano a cero (Hevesi et al. 1992).

c. Error cuadrático medio adimensional (ECMA):

$$
\mathbf{E C M A}=\frac{1}{n} \sum_{i=1}^{n} \frac{\left[z^{*}\left(x_{i}\right)-z\left(x_{i}\right)\right]^{2}}{\sigma_{k}}
$$

dónde: k es la desviación estándar del error esperado en la estimación con el krigeado. La validez del modelo se satisface si ECMA está comprendido entre los valores $1 \pm 2(2 / \mathrm{N}) 0.5$. d. Otro estadístico para validar el ajuste del modelo consiste en que el valor de la varianza de los errores sea menor a la varianza muestral.

Nivel de dependencia espacial. La determinación del grado de relación entre los datos o nivel de dependencia espacial se obtuvo al dividir el efecto pepita entre la meseta y expresando en porcentaje su resultado. Si el resultado es menor de $25 \%$ el nivel de dependencia espacial es alta, si se encuentra entre 26 y $75 \%$ el nivel es moderado y bajo si es mayor del $76 \%$ el nivel de dependencia (Cambardella et al. 1994).

Elaboración de mapas. Se realizó la interpolación de valores a través del krigeado ordinario que permite la estimación insesgada de valores asociados a puntos que no fueron muestreados, las estimaciones obtenidas fueron representadas en 
forma de mapa para cada fecha de muestreo mediante el uso del programa Surfer 9 (Surface Mapping System, Golden Software Inc., EE.UU.

\section{Resultados}

Las especies de trips identificados en las parcelas de estudio fueron, Frankliniella occidentalis Pergande (45\%), Scirtothrips perseae Nakahara (30\%) y Neohydatothrips signifer Priesner (25\%).

La distribución espacial que se obtuvo en las poblaciones de trips fue de tipo agregada, los modelos a los que se ajustaron los semivariogramas fueron esféricos en el caso de la parcela 1 (Tabla 1). La media de la población de trips en la parcela 1 varió de 5,09 individuos en mayo a 476,99 individuos en febrero (Tabla 2). En la parcela 2, de igual manera que en la 1 , los semivariogramas se ajustaron al modelo esférico (Tabla 3). Esto es indicativo que dentro de todas las parcelas analizadas se encuentran zonas en que hay una elevada manifestación de trips respecto al resto de puntos muestreados, es decir, existen focos de agregación de la plaga que avanzan desde una fuente puntual. Adicionalmente, la densidad media fluctuó entre 6,88 en julio a 504,78 individuos en febrero (Tabla 4). Las densidades más bajas se presentaron en mayo, mientras que las más altas en febrero, ya que en ese mes la planta de aguacate aún presenta floración y es en esa etapa fenológica donde encontramos mayor cantidad de trips.

Para todos los modelos ajustados se presentó un efecto pepita igual a cero, dicho valor es indicativo de que la escala de muestreo utilizada fue la correcta y el error de muestreo fue mínimo. Los valores del rango de la parcela 1 fueron de $14,4 \mathrm{~m}$ a $67,8 \mathrm{~m}$ (Tabla 1$)$, mientras que para la segunda par- cela los valores fluctuaron de 13,2 a 46,5 m (Tabla 3), siendo el rango la distancia máxima hasta la cual existe relación espacial entre los datos. Todos los modelos presentaron un alto nivel de dependencia espacial para cada una de las fechas de muestreo. Los modelos de la distribución espacial de trips en las parcelas estudiadas se validaron con los parámetros estadísticos al ubicarse dentro del rango permisible.

Los mapas de la superficie infestada de la parcela 1 en los meses de enero muestreo 1, abril muestreo 1, mayo muestreo 1 y 2 , agosto muestreo 2 y octubre muestreo 1 y 2 , indicaron que los focos de infestación estuvieron ubicados más hacia los bordes superior e inferior de la parcela; para enero 2, julio 1 y 2 , agosto 1 , marzo 1 y 2 , abril 2 y septiembre 1 los focos de infestación abarcaron la mayor parte de la parcela, mientras que en junio 1 y 2 , febrero 1 y 2 y septiembre 2 , los puntos de infestación se encontraron en la parte central de la parcela (Fig. 1).

Para la parcela 2 los meses de enero 1, febrero 2, marzo 1, julio 2, agosto 1 y 2 , septiembre 1 , y octubre 1 y 2 los centros de agregación de trips estuvieron localizados hacia la zona central de la parcela. Para marzo 2, abril 1 y 2 , mayo 1 y 2 , junio 1 y 2 y julio 1 la tendencia de los focos de agregación fue de ubicarse hacia los bordes de la parcela, mientras que en enero 2 , febrero 1 y septiembre 2 , los focos de infestación del trips estuvieron distribuidos en toda la zona de la parcela. En todos los meses de muestreo hubo presencia de trips, sin embargo, en febrero, marzo y agosto, se encontraron las mayores densidades de trips (Fig. 2).

En ambas parcelas se observó que en la mayoría de los meses la infestación fue del $100 \%$, sin embargo, en la parcela 1 hubo una ligera disminución en junio y octubre con un porcentaje de $92 \%$ y $88 \%$, respectivamente, mientras que para

Tabla 1. Parámetros (efecto pepita, meseta y rango) de los modelos ajustados a los semivariogramas de trips en la parcela 1.

\begin{tabular}{|c|c|c|c|c|c|c|c|}
\hline Fecha & Muestreos & Modelo & Pepita & Meseta & Rango & $\begin{array}{l}\text { Pepita / } \\
\text { Meseta }\end{array}$ & $\begin{array}{c}\text { Nivel de } \\
\text { dependencia } \\
\text { espacial }\end{array}$ \\
\hline \multirow{2}{*}{ Enero 2015} & Muestreo 1 & Esférico & 0 & 21,56 & 14,4 & 0 & Alta \\
\hline & Muestreo 2 & Esférico & 0 & 347,80 & 15,6 & 0 & Alta \\
\hline \multirow{2}{*}{ Febrero 2015} & Muestreo 1 & Esférico & 0 & 7372,00 & 54,4 & 0 & Alta \\
\hline & Muestreo 2 & Esférico & 0 & 2880,00 & 43,2 & 0 & Alta \\
\hline \multirow{2}{*}{ Marzo 2015} & Muestreo 1 & Esférico & 0 & 1564,00 & 46,4 & 0 & Alta \\
\hline & Muestreo 2 & Esférico & 0 & 1068,00 & 44,8 & 0 & Alta \\
\hline \multirow{2}{*}{ Abril 2015} & Muestreo 1 & Esférico & 0 & 118,90 & 46,4 & 0 & Alta \\
\hline & Muestreo 2 & Esférico & 0 & 30,80 & 44,8 & 0 & Alta \\
\hline \multirow{2}{*}{ Mayo 2015} & Muestreo 1 & Esférico & 0 & 40,42 & 43,2 & 0 & Alta \\
\hline & Muestreo 2 & Esférico & 0 & 10,20 & 44,8 & 0 & Alta \\
\hline \multirow{2}{*}{ Junio 2015} & Muestreo 1 & Esférico & 0 & 15,66 & 54,4 & 0 & Alta \\
\hline & Muestreo 2 & Esférico & 0 & 93,50 & 54,4 & 0 & Alta \\
\hline \multirow{2}{*}{ Julio 2015} & Muestreo 1 & Esférico & 0 & 206,80 & 65,6 & 0 & Alta \\
\hline & Muestreo 2 & Esférico & 0 & 372,00 & 65,6 & 0 & Alta \\
\hline \multirow{2}{*}{ Agosto 2015} & Muestreo 1 & Esférico & 0 & 1116,00 & 65,6 & 0 & Alta \\
\hline & Muestreo 2 & Esférico & 0 & 524,40 & 67,2 & 0 & Alta \\
\hline \multirow{2}{*}{ Septiembre 2015} & Muestreo 1 & Esférico & 0 & 213,20 & 46,4 & 0 & Alta \\
\hline & Muestreo 2 & Esférico & 0 & 169,10 & 25,6 & 0 & Alta \\
\hline \multirow{2}{*}{ Octubre 2015} & Muestreo 1 & Esférico & 0 & 64,08 & 25,6 & 0 & Alta \\
\hline & Muestreo 2 & Esférico & 0 & 43,64 & 67,8 & 0 & Alta \\
\hline
\end{tabular}


Tabla 2. Valores de los estadísticos de la validación cruzada de los semivariogramas obtenidos en los muestreos en la parcela 1: media de los errores de estimación (MEE), error cuadrático medio (ECM) y error cuadrático medio adimensional (ECMA).

\begin{tabular}{|c|c|c|c|c|c|c|c|}
\hline Fecha & Muestreos & $\begin{array}{c}\text { Media } \\
\text { Muestral }\end{array}$ & $\begin{array}{l}\text { Varianza } \\
\text { Muestral }\end{array}$ & MEE & $\begin{array}{l}\text { Varianza de } \\
\text { los errores }\end{array}$ & ECM & ECMA \\
\hline \multirow{2}{*}{ Enero 2015} & Muestreo 1 & 32,54 & 21,80 & $0,11 \mathrm{~ns}$ & 5,09 & 0,12 & 1,12 \\
\hline & Muestreo 2 & 128,36 & 369,70 & $0,07 \mathrm{~ns}$ & 36,10 & 0,09 & 1,10 \\
\hline \multirow{2}{*}{ Febrero 2015} & Muestreo 1 & 357,30 & 7556,6 & $0,13 \mathrm{~ns}$ & 44,22 & 0,12 & 1,08 \\
\hline & Muestreo 2 & 476,99 & 2992,51 & $0,11 \mathrm{~ns}$ & 37,19 & 0,10 & 1,10 \\
\hline \multirow{2}{*}{ Marzo 2015} & Muestreo 1 & 231,52 & 1693,32 & $0,08 \mathrm{~ns}$ & 28,93 & 0,07 & 1,07 \\
\hline & Muestreo 2 & 128,36 & 1162,14 & $0,12 \mathrm{~ns}$ & 51,94 & 0,11 & 1,13 \\
\hline \multirow{2}{*}{ Abril 2015} & Muestreo 1 & 42,14 & 131,15 & $0,10 \mathrm{~ns}$ & 20,16 & 0,13 & 1,10 \\
\hline & Muestreo 2 & 20,08 & 34,29 & $0,09 \mathrm{~ns}$ & 10,07 & 0,12 & 1,12 \\
\hline \multirow{2}{*}{ Mayo 2015} & Muestreo 1 & 14,57 & 46,36 & $0,12 \mathrm{~ns}$ & 5,99 & 0,09 & 1,09 \\
\hline & Muestreo 2 & 5,09 & 11,51 & $0,08 \mathrm{~ns}$ & 3,06 & 0,12 & 1,11 \\
\hline \multirow{2}{*}{ Junio 2015} & Muestreo 1 & 7,27 & 17,56 & $0,14 \mathrm{~ns}$ & 4,11 & 0,10 & 1,06 \\
\hline & Muestreo 2 & 27,37 & 102,71 & $0,07 \mathrm{~ns}$ & 27,85 & 0,08 & 1,10 \\
\hline \multirow{2}{*}{ Julio 2015} & Muestreo 1 & 41,50 & 215,72 & $0,10 \mathrm{~ns}$ & 29,06 & 0,10 & 1,13 \\
\hline & Muestreo 2 & 75,58 & 393,97 & $0,12 \mathrm{~ns}$ & 32,51 & 0,11 & 1,09 \\
\hline \multirow{2}{*}{ Agosto 2015} & Muestreo 1 & 145,36 & 1145,69 & $0,08 \mathrm{~ns}$ & 35,56 & 0,08 & 1,11 \\
\hline & Muestreo 2 & 86,95 & 569,39 & $0,12 \mathrm{~ns}$ & 47,04 & 0,11 & 1,12 \\
\hline \multirow{2}{*}{ Septiembre 2015} & Muestreo 1 & 57,37 & 257,89 & $0,10 \mathrm{~ns}$ & 35,28 & 0,07 & 1,07 \\
\hline & Muestreo 2 & 46,20 & 180,49 & $0,09 \mathrm{~ns}$ & 29,04 & 0,10 & 1,13 \\
\hline \multirow{2}{*}{ Octubre 2015} & Muestreo 1 & 18,12 & 71,14 & $0,07 \mathrm{~ns}$ & 8,15 & 0,12 & 1,10 \\
\hline & Muestreo 2 & 10,07 & 44,39 & $0,13 \mathrm{~ns}$ & 6,31 & 0,11 & 1,09 \\
\hline
\end{tabular}

Tabla 3. Parámetros (efecto pepita, meseta y rango) de los modelos ajustados a los semivariogramas de trips en la parcela 2.

\begin{tabular}{|c|c|c|c|c|c|c|c|}
\hline Fecha & Muestreos & Modelo & Pepita & Meseta & Rango & $\begin{array}{l}\text { Pepita / } \\
\text { Meseta }\end{array}$ & $\begin{array}{c}\text { Nivel de } \\
\text { dependencia } \\
\text { espacial }\end{array}$ \\
\hline \multirow{2}{*}{ Enero 2015} & Muestreo 1 & Esférico & 0 & 51,30 & 13,2 & 0 & Alta \\
\hline & Muestreo 2 & Esférico & 0 & 408,90 & 13,5 & 0 & Alta \\
\hline \multirow{2}{*}{ Febrero 2015} & Muestreo 1 & Esférico & 0 & 3485,00 & 46,5 & 0 & Alta \\
\hline & Muestreo 2 & Esférico & 0 & 21250,00 & 43,5 & 0 & Alta \\
\hline \multirow{2}{*}{ Marzo 2015} & Muestreo 1 & Esférico & 0 & 3610,00 & 27,0 & 0 & Alta \\
\hline & Muestreo 2 & Esférico & 0 & 1530,00 & 43,5 & 0 & Alta \\
\hline \multirow{2}{*}{ Abril 2015} & Muestreo 1 & Esférico & 0 & 171,00 & 39,0 & 0 & Alta \\
\hline & Muestreo 2 & Esférico & 0 & 63,90 & 42,0 & 0 & Alta \\
\hline \multirow{2}{*}{ Mayo 2015} & Muestreo 1 & Esférico & 0 & 57,42 & 40,5 & 0 & Alta \\
\hline & Muestreo 2 & Esférico & 0 & 29,05 & 43,5 & 0 & Alta \\
\hline \multirow{2}{*}{ Junio 2015} & Muestreo 1 & Esférico & 0 & 32,30 & 42,0 & 0 & Alta \\
\hline & Muestreo 2 & Esférico & 0 & 9,12 & 42,0 & 0 & Alta \\
\hline \multirow{2}{*}{ Julio 2015} & Muestreo 1 & Esférico & 0 & 9,57 & 40,5 & 0 & Alta \\
\hline & Muestreo 2 & Esférico & 0 & 32,34 & 42,0 & 0 & Alta \\
\hline \multirow{2}{*}{ Agosto 2015} & Muestreo 1 & Esférico & 0 & 238,00 & 40,5 & 0 & Alta \\
\hline & Muestreo 2 & Esférico & 0 & 800,40 & 39,0 & 0 & Alta \\
\hline \multirow{2}{*}{ Septiembre 2015} & Muestreo 1 & Esférico & 0 & 483,80 & 42,0 & 0 & Alta \\
\hline & Muestreo 2 & Esférico & 0 & 156,60 & 39,0 & 0 & Alta \\
\hline \multirow{2}{*}{ Octubre 2015} & Muestreo 1 & Esférico & 0 & 240,00 & 40,5 & 0 & Alta \\
\hline & Muestreo 2 & Esférico & 0 & 156,40 & 43,5 & 0 & Alta \\
\hline
\end{tabular}



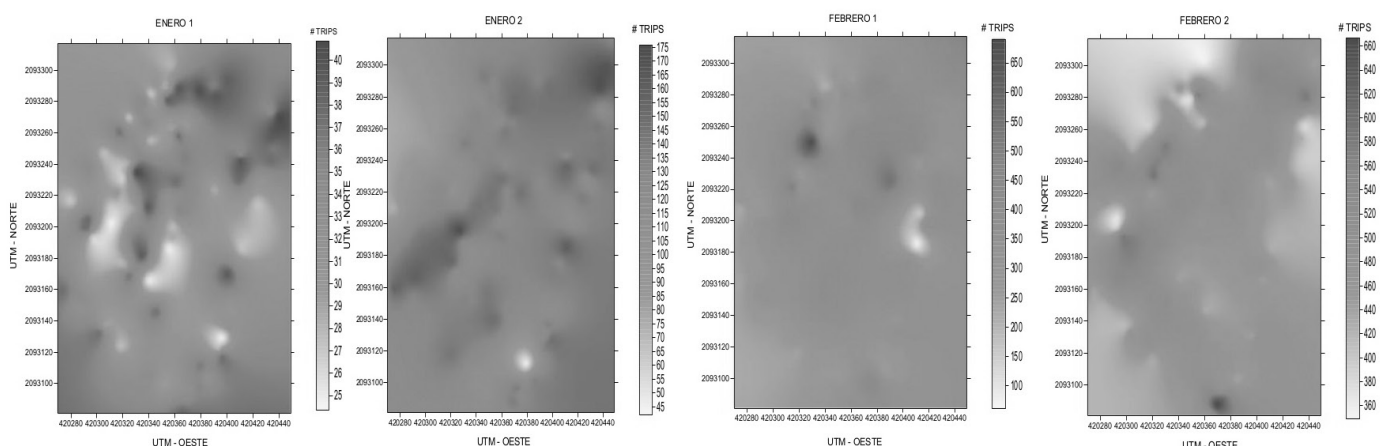

MARZO 1

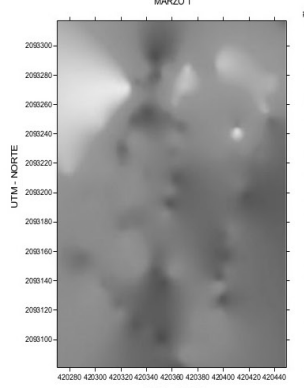

UTM- -

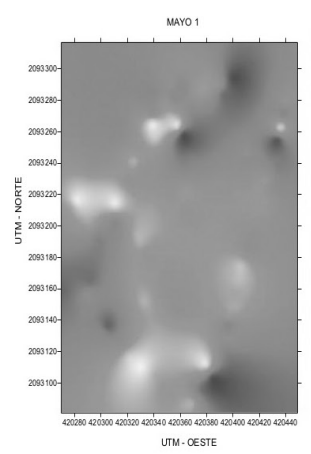

งul10 1

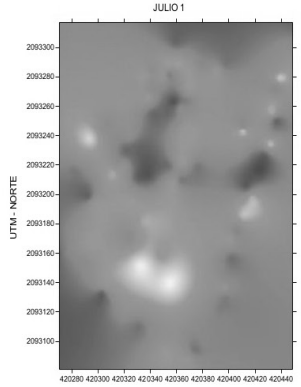

UTM- СESTE

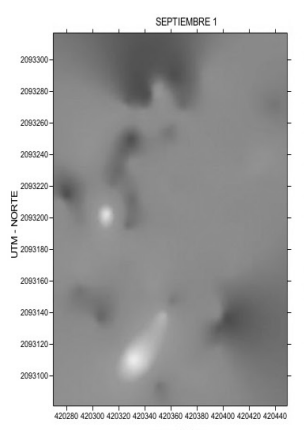

UTM. CESTE

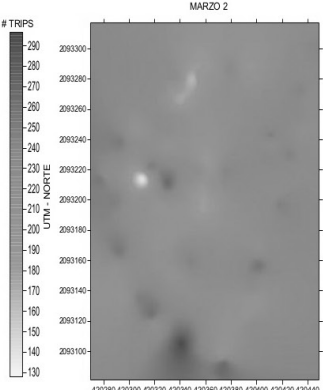

UTV. CESTE
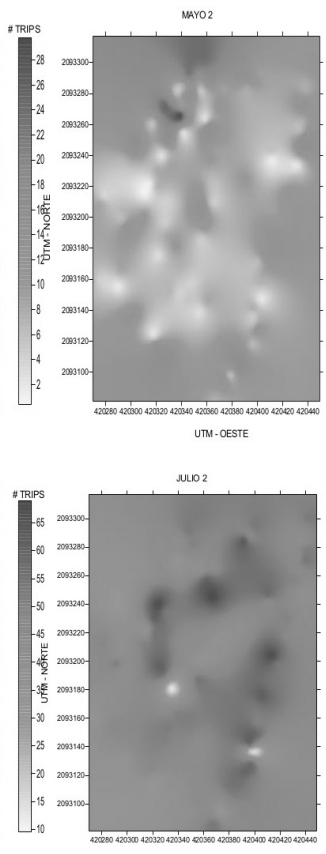

UTM- OESTE

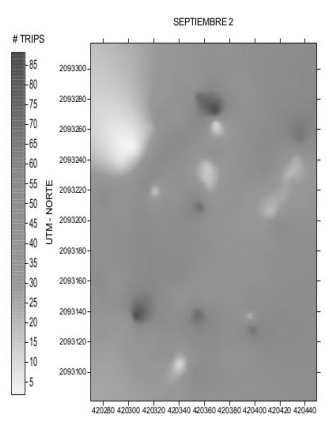

UTM-OESTE
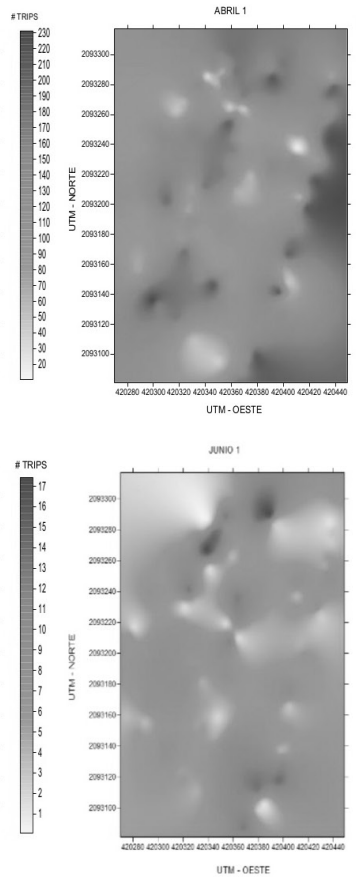

AGosto

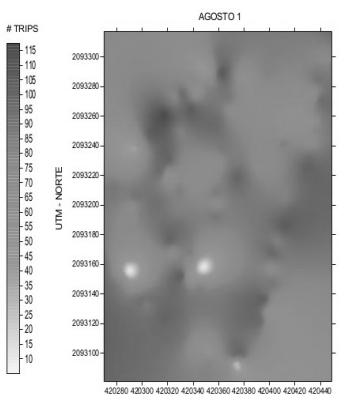

UTM-OESTE

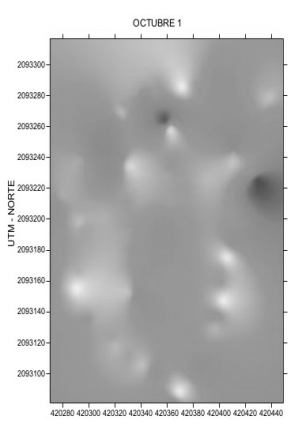

UTM-OESTE

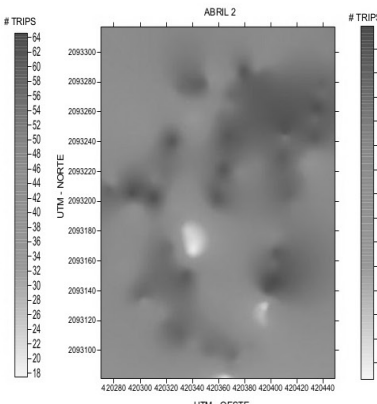

Junio2

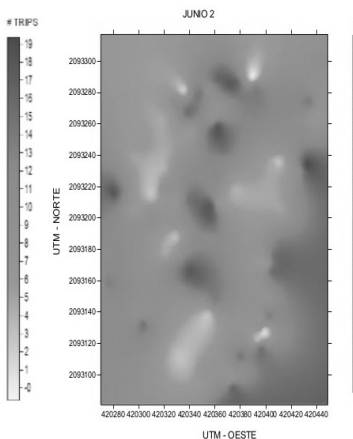

ACOSTO2

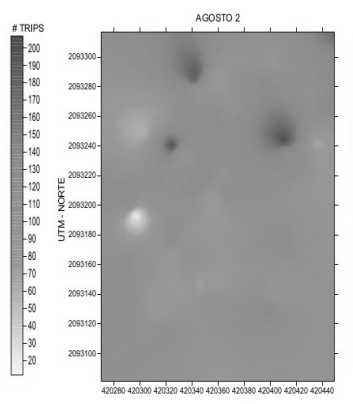

UTM-OESTE

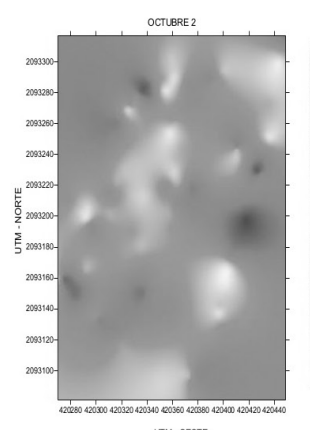

Figura 1. Mapas de densidad de las poblaciones de trips obtenidos en los muestreos de la parcela 1 en la localidad San José, Coatepec Harinas, México. 

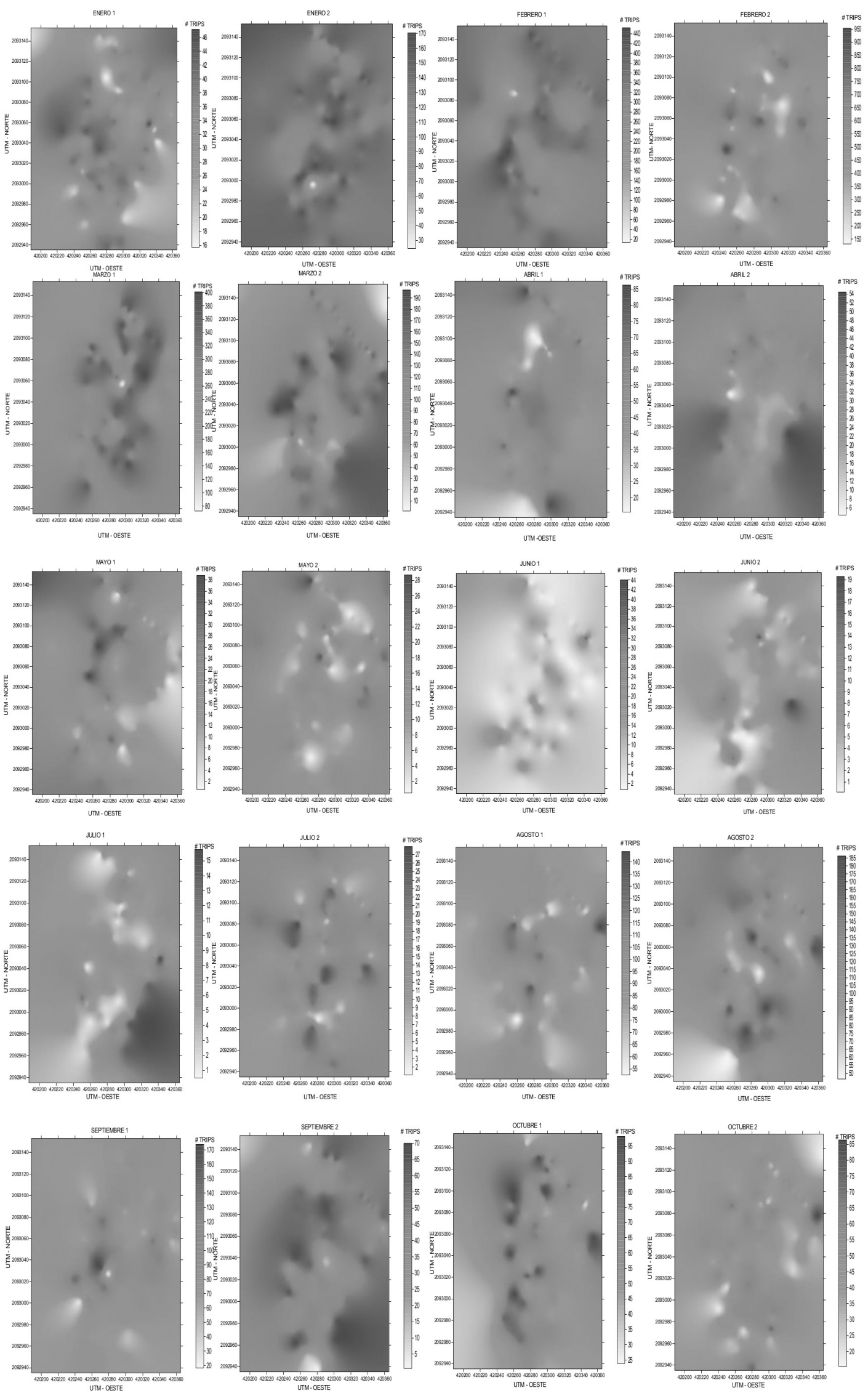

Figura 2. Mapas de densidad de las poblaciones de trips obtenidos en los muestreos de la parcela 2 en la localidad San Martín, Coatepec Harinas, México. 
Tabla 4. Valores de los estadísticos de la validación cruzada de los semivariogramas obtenidos en los muestreos en la parcela 2 : media de los errores de estimación (MEE), error cuadrático medio (ECM) y error cuadrático medio adimensional (ECMA).

\begin{tabular}{|c|c|c|c|c|c|c|c|}
\hline Fecha & Muestreos & $\begin{array}{c}\text { Media } \\
\text { muestral }\end{array}$ & $\begin{array}{l}\text { Varianza } \\
\text { muestral }\end{array}$ & MEE* & $\begin{array}{l}\text { Varianza de } \\
\text { los errores }\end{array}$ & ECM & ECMA \\
\hline \multirow{2}{*}{ Enero 2015} & Muestreo 1 & 32,32 & 53,10 & $0,11 \mathrm{~ns}$ & 12,61 & 0,13 & 1,10 \\
\hline & Muestreo 2 & 131,25 & 469,82 & $0,07 \mathrm{~ns}$ & 37,99 & 0,09 & 1,06 \\
\hline \multirow{2}{*}{ Febrero 2015} & Muestreo 1 & 320,68 & 4005,86 & $0,14 \mathrm{~ns}$ & 68,25 & 0,12 & 1,12 \\
\hline & Muestreo 2 & 504,78 & 24629,00 & $0,10 \mathrm{~ns}$ & 32,07 & 0,10 & 1,08 \\
\hline \multirow{2}{*}{ Marzo 2015} & Muestreo 1 & 284,23 & 3782,44 & $0,12 \mathrm{~ns}$ & 49,30 & 0,07 & 1,12 \\
\hline & Muestreo 2 & 121,14 & 1785,71 & $0,08 \mathrm{~ns}$ & 37,12 & 0,12 & 1,09 \\
\hline \multirow{2}{*}{ Abril 2015} & Muestreo 1 & 51,02 & 185,35 & $0,09 \mathrm{~ns}$ & 28,65 & 0,13 & 1,12 \\
\hline & Muestreo 2 & 29,60 & 70,59 & $0,11 \mathrm{~ns}$ & 15,72 & 0,10 & 1,07 \\
\hline \multirow{2}{*}{ Mayo 2015} & Muestreo 1 & 19,63 & 65,03 & $0,07 \mathrm{~ns}$ & 13,08 & 0,11 & 1,13 \\
\hline & Muestreo 2 & 11,47 & 34,68 & $0,13 \mathrm{~ns}$ & 9,64 & 0,13 & 1,10 \\
\hline \multirow{2}{*}{ Junio 2015} & Muestreo 1 & 7,30 & 37,84 & $0,10 \mathrm{~ns}$ & 7,51 & 0,10 & 1,09 \\
\hline & Muestreo 2 & 6,09 & 11,12 & $0,08 \mathrm{~ns}$ & 4,02 & 0,07 & 1,11 \\
\hline \multirow{2}{*}{ Julio 2015} & Muestreo 1 & 6,88 & 10,16 & $0,14 \mathrm{~ns}$ & 1,37 & 0,14 & 1,07 \\
\hline & Muestreo 2 & 14,28 & 32,40 & $0,12 \mathrm{~ns}$ & 4,69 & 0,10 & 1,14 \\
\hline \multirow{2}{*}{ Agosto 2015} & Muestreo 1 & 120,39 & 277,52 & $0,09 \mathrm{~ns}$ & 28,19 & 0,11 & 1,08 \\
\hline & Muestreo 2 & 90,68 & 865,66 & $0,11 \mathrm{~ns}$ & 56,04 & 0,08 & 1,13 \\
\hline \multirow{2}{*}{ Septiembre 2015} & Muestreo 1 & 46,77 & 585,48 & $0,07 \mathrm{~ns}$ & 28,14 & 0,13 & 1,10 \\
\hline & Muestreo 2 & 89,99 & 179,68 & $0,11 \mathrm{~ns}$ & 19,05 & 0,07 & 1,09 \\
\hline \multirow{2}{*}{ Octubre 2015} & Muestreo 1 & 64,80 & 295,01 & $0,10 \mathrm{~ns}$ & 27,69 & 0,05 & 1,11 \\
\hline & Muestreo 2 & 45,57 & 164,73 & $0,08 \mathrm{~ns}$ & 31,06 & 0,11 & 1,12 \\
\hline
\end{tabular}

el caso de la parcela 2 en todos los muestreos la infestación alcanzó el $100 \%$ de las parcelas, pero con diferentes densidades poblacionales.

\section{Discusión}

Se registraron trips en las dos parcelas de aguacate, sin embargo, la mayor densidad de poblaciones de trips en ambas parcelas se presentó en febrero, marzo y agosto; esto debido a que los árboles se encontraban en etapa de floración. González et al. (2000) registraron en Michoacán, poblaciones de trips con picos entre marzo y mayo, mientras que durante el periodo de lluvias (junio-septiembre) la población bajo, para aumentar de nuevo a partir de octubre; esto fue en huertas de la variedad Hass con fruto bien definido de tamaño pequeño. Solares et al. (2013) al realizar un estudio de distribución espacial de trips en aguacate en Zitácuaro, Michoacán encontraron que la mayor densidad de trips se presentó desde julio a septiembre. Las poblaciones de trips bajan durante el periodo de lluvias y para agosto empiezan a aumentar sus poblaciones.

La observación de la estructura agregada en la distribución espacial de trips en plantaciones de aguacate se hizo con el uso de la geoestadística. En comparación con la estimación de la distribución espacial que realiza la estadística clásica, los modelos geoestadísticos proporcionan una medida más directa de la dependencia espacial, ya que tienen en cuenta la naturaleza bidimensional de la distribución de los organismos a través de su exacta localización espacial y es independiente de la relación entre la media y la varianza (Samper y Carrera 1996). La geoestadística además permite elaborar mapas de densidad de la distribución espacial de un organismo (Rossi et al. 1992; Ribes et al. 1998). García
(2004) utilizó la geoestadística para estudiar la distribución espacial del lepidóptero Helicoverpa armigera Hübner, en una parcela de tomate, con el empleo de la función variograma, obteniendo más representaciones gráficas diarias de dichas distribuciones. Estos mapas se basan en la técnica de estimación geoestadística conocida como "krigeado", particularmente el ordinario en bloques. Contreras et al. (2016) realizaron un estudio de distribución espacial de la enfermedad Candidatus liberibacter solanacearum y de su vector Bactericera cockerelli Sulc en papa, en el cual pudieron obtener mapas de densidad de B. cockerelli tanto en huevos, ninfas y adultos, usando el krigeado.

El uso de las técnicas para modelizar la distribución espacial de insectos ha mostrado ser una herramienta muy eficiente para determinar la distribución espacial de diversos insectos plaga en cultivos de importancia económica. Diferentes trabajos como el de Jiménez et al. (2000) revelan la distribución temporal y agregada de thrips palmi Karny en papa en Cuba. Por otra parte, Sciarretta et al. (2008) encontraron una distribución agregada con ajuste al modelo esférico en Lobesia botrana en parcelas de uva. En cuanto a Bressan et al. (2010) determinaron la distribución espacial de Pentastiridius leporinus L. en campos de trigo; Ramírez et al. (2011) la distribución agregada de Bactericera cockerelli en papa y Jiménez et al. (2013) las de poblaciones de trips en tomate de cáscara con distribución agregada con varios centros de agregación visualizados en los mapas obtenidos y sus semivariogramas de la distribución espacial se ajustaron a los modelos esférico y gaussiano. De la misma forma que Quiñones-Valdez et al. (2015) en todo el ciclo fenológico del cultivo del gladiolo, generando mapas que reflejaron la estructura agregada de la plaga en puntos específicos dentro de 
la parcela. Contrario a esto Carrillo et al. (2004) realizaron un estudio de la distribución espacial de larvas de escarabaeidos en plantas cultivadas y sus resultados mostraron que las larvas estudiadas no tenían una distribución agregada, esto pudo haber estado relacionado con la amplia polifagia de las larvas. Estas técnicas también se han aplicado en enfermedades como el carbón de la espiga del maíz (Sánchez et al. 2011) y han permitido describir la distribución en agregados de los insectos plaga y enfermedades mencionados, determinando dicha agregación en puntos específicos en las parcelas y regiones estudiadas.

El comportamiento espacial de las poblaciones de trips de forma agregada en las parcelas de aguacate, permite sugerir que se pueden manejar $\mathrm{y}$, por lo tanto, reducirlas aplicando y dirigiendo medidas de control hacia los centros de agregación o puntos específicos en los que se encuentra la plaga, evitando aplicaciones generalizadas o de cobertura total, lo cual nos lleva a un ahorro en los insumos, visualizando los focos de infestación a través de los mapas generados. Lo anterior concuerda con Rong et al. (2006) y Esquivel y Jasso (2014) quienes, respectivamente, propusieron el manejo de Locusta migratoria manilensis Linnaeus y del gusano soldado en el cultivo de maíz en puntos específicos de presencia de la plaga.

Los valores de cero encontrados en el efecto pepita en todos los modelos ajustados, permite afirmar que la escala de muestreo utilizada fue la adecuada y el error de muestreo fue mínimo (Oliver y Webster 1998), esto sugiere que los modelos ajustados tienen un $98 \%$ de confiabilidad. En consecuencia, es válido deducir que más del $90 \%$ de la variación total es debido a la dependencia espacial en la escala de muestreo utilizada; dicho de otra manera, más del $90 \%$ de la variación de la distribución de las poblaciones del trips se logró explicar por la estructura espacial establecida con los semivariogramas (Liebhold y Sharov 1998). Ramírez et al. (2011) encontraron valores de pepita igual a cero en un estudio de la distribución espacial de Bactericera cockerelli en papa. Valores de pepita iguales fueron obtenidos por Solares et al. (2013) en la distribución espacial de trips en plantaciones comerciales de aguacate.

La distribución espacial ajustada al modelo esférico es indicativo que dentro de la parcela analizada existen zonas en que se manifiesta más la plaga respecto al resto de puntos considerados en el muestreo y, que se desplaza de un punto específico hacia otros puntos dentro de la parcela, estos puntos de agregación presentan un crecimiento rápido cerca de su origen pero los incrementos van decreciendo conforme aumenta la distancia, esto es factible dado el comportamiento del trips, los focos de infestación se observan en los mapas obtenidos. Moral García (2003) al realizar la distribución espacial de $H$. armigera y sus variogramas teóricos, encontró que se ajustaron a modelos esféricos o exponenciales, con un efecto pepita. Contreras et al. (2016) encontraron que los semivariogramas para huevos y ninfas de $B$. cockerelli se ajustaron al modelo esférico, lo que indicó la agregación de la plaga en ciertas zonas de la parcela.

La distribución de los trips presentó un alto nivel de dependencia espacial en todos los modelos ajustados, esto es indicativo de la existencia de una fuerte relación espacial de trips en los puntos muestreados. El alto nivel de dependencia espacial resultó de dividir el efecto pepita entre la meseta que fue menor a $25 \%$ para todos los semivariogramas. Los valores del efecto pepita indicaron una alta dependencia espacial lo cual sugiere que las poblaciones de trips dependen entre sí y que el nivel de agregación es alto (Rossi et al. 1992). Esquivel y Jasso (2014) en un estudio de la distribución espacial de gusano soldado en seis localidades del Estado de México, utilizando las técnicas de la geoestadística, mostraron la existencia de una estructura espacial agregada, con un nivel de dependencia espacial alto para las seis localidades en estudio. En los mapas de densidad que se obtuvieron mediante la técnica del krigeado se observan los centros de agregación que presentó el insecto dentro de la parcela, lo cual a la hora de proteger el cultivo contra la plaga nos permite dirigir de forma precisa las medidas de control (Weisz et al. 1996; Fleischer et al. 1999a, b; Ribes et al. 1998; García 2004; Rong et al. 2006; Esquivel y Jasso 2014). Esto ayudará a que los insectos no generen resistencia a los plaguicidas debido a la creación de refugios temporales dinámicos, al no tratar toda la superficie del cultivo (Moral et al. 2011). Con estas acciones se evitará una mayor distribución de los trips a ciertos niveles poblacionales y que puedan causar daños económicos mayores. El comportamiento de los focos de infestación dentro de la parcela permite suponer que los trips se originan de una fuente puntual (Hoddle 2002).

Los mapas permitieron identificar las áreas de infestación, encontrando que el trips se distribuyó en el $100 \%$ de la parcela, sin embargo, hay áreas en las que se encuentran poblaciones más altas, esto concuerda con Jiménez et al. (2008), quienes determinaron la distribución espacial y mapeo de Curculio elephas Gyllenhal en Quercus ilex Linneo, ellos elaboraron cuatro mapas de densidad, uno por cada año, utilizando el método de la distancia inversa como interpolador del programa Surfer 7; estos mapas indican que el insecto se localizó por toda la parcela con una distribución espacial agregada. Contrario a estos resultados QuiñonesValdez et al. (2015) encontraron que las poblaciones de trips en el cultivo del gladiolo no infestaron el $100 \%$ de la parcela y estos se encontraban en forma agregada en puntos específicos de infestación, Esquivel y Jasso (2014) realizaron un estudio de distribución espacial y mapeo de gusano soldado en seis localidades en el estado de México en maíz y encontraron que en ninguna de las localidades se presentó un área de infestación del $100 \%$ y el porcentaje máximo alcanzado fue del $94 \%$, Jiménez et al. (2013) reportaron que las poblaciones de F. occidentalis en tomate de cáscara no se distribuyen en la totalidad de las parcelas. Ramírez et al. (2013) encontraron que los mapas de infestación de B. cockerelli Sulc sobre papa reflejaron una estructura agregada del insecto el cual no invadió el $100 \%$ de la superficie de la parcela, permitiendo identificar áreas libres de infestación, Ramírez y Porcayo (2008) reportaron que las ninfas de $J a$ cobiasca lybica Bergenin y Zanon no invadieron el $100 \%$ de las parcelas de vid en estudio, por lo tanto, la infestación no fue uniforme.

\section{Conclusiones}

La distribución espacial de las poblaciones de trips se presenta en agregados en las parcelas de estudio. Dicha agregación se visualiza perfectamente en los mapas de densidad elaborados. Con la información obtenida en este trabajo se puede contribuir a establecer esquemas de muestreo más eficientes, para después establecer umbrales económicos y entonces proponer el inicio de acciones que ayuden a suprimir las poblaciones de trips en los huertos de aguacate e iniciar 
acciones solo cuando sea necesario, focalizando las medidas de acción sobre las áreas específicas de infestación, lo que conlleva un ahorro económico importante y una reducción significativa del impacto sobre el medio ambiente. Con las técnicas geoestadísticas fue posible obtener la distribución espacial y los mapas de densidad de las poblaciones de trips, los cuales se ubicaron en varios centros de agregación dentro del cultivo de aguacate en las parcelas estudiadas.

\section{Literatura citada}

ASCENSIÓN, B. G.; BRAVO, M. H.; GONZÁLEZ, H. H.; JOHANSEN, N. R. BECERRIL, R. A. 1999. Fluctuación poblacional y daño de trips en aguacate C.V. Hass. Revista Chapingo Serie Horticultura 5: 291-296.

BRESSAN, A.; MORAL, J. F.; SÉMÉTEY, O.; BOUDON, PADIEU, E. 2010. Spatio-temporal pattern of Pentastiridius leporinus migration in an ephemeral cropping system. Agricultural and Forest Entomology 12: 59-68.

CAMBARDELLA, C. A.; MOORMAN, T. B.; NOVAK, J. M.; PARKIN, T. B.; KARLEN, D. L.; TURCO, R. F.; KONOPKA, A. E 1994. Field scale variability of soil properties in central Iowa soils. Soil Science. Society American Journal 58: 1501-1511.

CARRILlO, R.; PAPE, H.; NEIRA, M.; BALOCCHI, O. 2004. Distribución espacial de larvas de dos especies de escarabaeidos nativos en respuesta a plantas cultivadas. Revista Chilena de Entomología 30 (1): 59-64.

CONTRERAS, R. A.; GUTIÉRREZ, I. A. T.; SILVA, R. H. V.; SÁNCHEZ, P. J. R.; LAGUNA, C. A.; RAMÍREZ, D. J. F. 2016. Distribución espacial de Candidatus Liberibacter solanacearum y Bactericera cockerelli (Sulc) (Hemiptera: Triozidae) en papa (Solanum tuberosum L.). Southwestern Entomologist 41 (1): 105-114.

CORIA, A. V. M. 1993. Principales plagas del aguacate en Michoacán Folleto para productores Núm. 19. SAGARPA INIFAP.

ENGLUND, E.; SPARKS, A. 1988. GEO-EAS (Geostatistical Environmental Assessment Software) User's guide. U.S. Environmental protection agency document EPA/600/4-88/033. Environmental Monitoring Systems Laboratory, Las Vegas, NV, EE.UU.

ESQUIVEL, H. V.; JASSO, G.Y. 2014. Distribución espacial y mapeo de gusano soldado en seis localidades del Estado de México, en el año 2011. Revista Mexicana de Ciencias Agrícolas 5: 923-935.

FISHER, J. B.; DAVENPORT, T. L. 1989. Structure and development of surface deformation on avocado fruits. HortScience 24: 841-844.

FLEISCHER, S. J.; BLOM, P. E.; WEISZ, R. 1999a. Sampling in precision IPM: When the objective is a map. The American Phytopathological Society 89 (11): 115-118.

FLEISCHER, S. J.; BLOM, P. E.; EMMEN, D.; HOWER, A. 1999b. Dynamics in the spatial continuity of insect density. Precision Agriculture, (precisionagric4a), pp. 133-141.

GARCÍA, F. M. 2004. Aplicación de la geoestadística en las ciencias ambientales. Revista Ecosistemas 13 (1): 78-86.

GONZÁLEZ, H.; SOSA, C.; GONZÁLEZ, M.; VALLE DE LA PAZ, A. 1998. Selección del color de trampas adhesivas para muestreo de trips en el cultivo de aguacate (Persea americana Mill.). pp. 209-210. En: Avances de investigación, 1997. Instituto de Fitosanidad, Colegio de Postgraduados. Montecillo, México.

GONZÁlEZ H., H.; JOHANSEN, R.; GASCA, L.; EQUIHUA, A.; SALINAS, A.; ESTRADA, E.; DURAN, F.; VALLE, A. 2000. Plagas del aguacate. pp. 177-186. En: Téliz, D. (cd). El aguacate y su manejo integrado. Ediciones Mundi Prensa. México, D.F.

HEVESI, J.; ISTOK, J.; FLINT, A. 1992. Precipitation estimation in mountainous terrain using multivariate geostatistics. Part. I.
Structural analysis. Journal of Applied Meteorology 31 (7): 661676.

HODDLE, M. S. 2002. Developmental and reproductive biology of Scirtothrips perseae (Thysanoptera: Thripidae): a new avocado pest in California. Bulletin of Entomological Research 92 (04): 279-285.

ISAAKS, E. H.; SRIVASTAVA, R. M. 1989. Spatial continuity measures for probabilistic and deterministic geostatistics. Mathematical Geology 20 (4): 313-341.

JIMÉNEZ, S. F.; CORTIÑAS, J.; LÓPEZ, D. 2000. Distribución temporal y espacial y consideraciones para el monitoreo de Thrips palmi en papa en Cuba. Manejo Integrado de Plagas (CATIE) 57: 54-57.

JIMÉNEZ, A.; LÓPEZ, M.; GONZÁLEZ, A. J.; OCETE, M.; SORIA, F. 2008. Distribución espacial y mapeo de Curculio elephas Gyllenhal en encina (Quercus ilex L.). Agrociencia 12: 35-43.

JIMÉNEZ, R. D. L. A.; RAMÍREZ, D. J. F.; SÁNCHEZ, P. J. R.; SALGADO, S. M. L.; LAGUNA, C. A. 2013. Modelización espacial de Frankliniella occidentalis (Thysanoptera: Thripidae) en tomate de cáscara por medio de técnicas geoestadísticas. Revista Colombiana de Entomología 39 (2): 183-192.

JOHANSEN, R. M.; MOJICA G. A.; ASCENCIÓN, B. G. 1999. Introducción al conocimiento de los insectos tisanópteros mexicanos, en el aguacatero (Persea americana Mill). Revista Chapingo Serie Horticultura 5: 279-285.

JOURNEL, A. G.; HUIJBREGTS, C. J. 1978. Mining geostatistics. Academic Press, Londres, Reino Unido 600 p.

LIEBHOLD, A. M.; SHAROV, A. A. 1998. Testing for correlation in the presence of spatial autocorrelation in insect count data. pp. 111-117. In: Baumgartner, J.; Brandmayr, P.; Manly, B. F. J. (Eds.). Population and community ecology for insect management and conservation. Balkema, Rotterdam.

MORAL GARCÍA, F. J. 2003. Analysis of the spatio-temporal distribution of Helicoverpa armígera $\mathrm{Hb}$. in a tomato field using a stochastic approach. Biosystems Engineering 93 (3): 253-259.

MORAL, F. J.; TERRON, J. M.; REBOLLO, F. J. 2011. Site-specific management zones based on the Rasch model and geostatistical techniques. Computers and Electronic in Agriculture 75: 223-230.

NAAMANI, G. 2007. Developments in the avocado world. California Avocado Society pp. 71-76.

OLIVER, M.; WEBSTER, R. 1998. How geostatistics can help you. Soil Use and Management 7 (4): 206-217.

QUIÑONES-VALDEZ R.; SÁNCHEZ-PALE J. R.; PEDRAZAESQUIVEL, A. K.; CASTAÑEDA-VILDOZOLA A.; GUTIERREZ-IBAÑEZ, A. T.; RAMÍREZ-DÁVILA, J. F. 2015. Análisis espacial de Thrips spp. (Thysanoptera) en el cultivo de gladiolo en la región sureste del Estado de México, México. Southwestern Entomologist 40 (2): 397-408.

RAMÍREZ, D. J. F.; PORCAYO, C. E. 2008. Distribución espacial de las ninfas de Jacobiasca lybica (Hemiptera: Cicadellidae) en un viñedo en Andalucia, España. Revista Colombiana de Entomología 34 (2): 169-175.

RAMÍREZ, D. J. F.; PORCAYO, C. E.; SÁNCHEZ, P. J. R. 2011. Análisis de la distribución espacial de Bactericera cockerelli Sulc (Hemiptera: Triozidae) en Solanum tuberosum L. en Donato Guerra, México. Boletín del Museo de Entomología de la Universidad del Valle 12 (1): 12-24.

RAMÍREZ, D. J. F.; SOLARES, A. V. M.; FIGUEROA, F. D. K.; SÁNCHEZ, P. J. R. 2013. Comportamiento espacial de trips (Insecta: Thysanoptera), en plantaciones comerciales de aguacate (Persea americana Mill.) en Zitácuaro, Michoacán, México. Acta Zoológica Mexicana 29 (3): 545-562.

RIBES, D. M.; BASCUÑANA, C. M.; AVILLA, H. J. 1998. Estudio de la distribución espacial de Cydia pomonella (L.) y Pandemis heparana (Denis \& Schiffermüller) en Torregrossa (Lleida) mediante métodos geoestadísticos. Boletín de Sanidad Vegetal Plagas 24 (4): 935-948. 
RONG, J.; DIAN, M. L.; BAO, Y. X.; ZHE, L.; DONG, L. 2006. Spatial distribution of oriental migratory locust (Orthoptera: Acrididae) egg pod populations: Implications for site-specific pest management. Environmental Entomology 35: 1244-1248.

ROSSI, R. E.; MULLA, J. D.; JOURNEL, A. G.; FRANZ, E. H. 1992. Geostatical tools for modeling and interpreting ecological spatial dependence. Ecological Monographs 62 (2): 277-314.

SALGADO, S. M. L. 1993. Problemas fitosanitarios del aguacate en Coatepec Harinas. pp. 191-212. En: Memorias Centro de Investigaciones científicas y tecnológicas del aguacate en el Estado de México.

SAMPER, F. J.; CARRERA, J. 1996. Geoestadística, aplicaciones a la hidrogeología. Centro Internacional de Métodos Numéricos en Ingeniería, España, p. 484.

SANCHEZ-PALE, J. R.; RAMIREZ DAVILA, J. F.; GONZALEZHUERTA, H. A.; DE LEON, C. 2011. Modelization of the spatial distribution of corn head smut (Sporisorium reilianum Langdon and Fullerton) in Mexico. Spanish Journal of Agricultural Research 9 (3): 882-893.

SCIARRETTA, A.; ZINNI, A.; MAZZOCCHETTI, A.; TREMATERRA, P. 2008. Spatial analysis of Lobesia botrana (Lepidoptera: Tortricidae) male population in a Mediterranean agricultural landscape in central Italy. Environmental Entomology 37 (2): 382-390.
SIAP. 2014. Servicio de Información Agroalimentaria y Pesquera. SIAP. http://www.siap.gob.mx/ (Última consulta: 7 noviembre 2014).

SOLARES, A. V. M.; RAMÍREZ, D. J. F.; SÁNCHEZ, P. J. R. 2013. Distribución Espacial de trips (Insecta: Thysanoptera) en el cultivo de aguacate (Persea americana Mill.). Boletín del Museo de Entomología de la Universidad del Valle 12 (2): 1-12.

WEISZ, R.; FLEISCHER, J.; SMILOWITZ, Z. 1996. Site specific integrated pest management for high value crops: Impact on potato pest management. Journal of Economic Entomology 89: 501-509.

Recibido: 28-may-2016 • Aceptado: 12-jul-2017

Citación sugerida:

RIVERA-MARTÍNEZ，R.; RAMÍREZ-DÁVILA，J. F.; RUBÍARRIAGA, M.; DOMÍNGUEZ-LÓPEZ, A.; ACOSTA-GUADARRAMA, A. D.; FIGUEROA-FIGUEROA, D. K. 2017. Modelización espacial de trips (Insecta: Thysanoptera) en el cultivo de aguacate (Persea americana). Revista Colombiana de Entomología 43 (2): 131-140. Julio - Diciembre 2017. ISSN 0120-0488. 